\section{PTU-209 IS UNSEDATED COLONOSCOPY THE WAY FORWARD?}

doi:10.1136/gutjnl-2012-302514c.209

J Addley, ${ }^{*}$ M Mitchell, S Johnston, I Mainie. Gastroenterology, Belfast City Hospital, Belfast, UK

Introduction Colonoscopy is a very common procedure however, unsedated colonoscopy remains underused. The use of sedation increases adverse events, prolongs recovery, affects ability to recall information relating to procedure outcome, and can impact on the efficiency of an endoscopy unit.

Methods A retrospective analysis was carried out on patients attending a large teaching hospital for outpatient colonoscopy from September 1st 2009 to December 31st 2010. A proforma was completed with details relating to demographics, seniority of endoscopist, presence of a trainee, reason for referral, sedation use, outcome of procedure, interventions required, subsequent complications and comfort scores.

Results 244 patients had unsedated colonoscopies (68 female and 176 male) with a median age of 60.6 years. These were matched with 244 randomly selected colonoscopies during that time period. The completion rate was $96 \%$ in the unsedated group and $91 \%$ in the sedated group $(\mathrm{p}<0.041)$. The mean comfort score in the unsedated colonoscopy group was 1.93 and 1.79 in the sedated group. Mean procedure time was $23.6 \mathrm{~min}$ (sedated) and $22 \mathrm{~min}$ (unsedated). There were no immediate complications in the unsedated group. Complications in the sedated group were seen in 5: Vasovagal episode $(n=1)$, repiratory depression $(n=2)$, bleeding post polypectomy $(\mathrm{n}=1)$.

Conclusion An increasing number of unsedated colonoscopies are being performed successfully in our unit with high completion rates, shorter procedure time and similar comfort scores between sedated and unsedated groups. A heightened awareness of the availability of unsedated colonoscopy is required-and it should be offered to all suitable patients.

Competing interests None declared.

\section{REFERENCES}

1. Petrini JL, Egan JV, Hahn WV. Unsedated colonoscopy: patient characteristics and satisfaction in a community-based endoscopy unit. Gastrointest Endosc 2009; 69:567-72

2. Ko CW, Riffle S, Michaels L, et al. Serious complications within 30 days of screening and surveillance colonoscopy are uncommon. Clin Gastro Hepatol 2010; 8:166-73.

3. Fisher DA, Maple JT, Ben-Menachem T, et al. ASGE standards of practice committee complications of colonoscopy. Gastrointest Endosc 2011:74:745-52.

4. British Society of Gastroenterology. Guidelines on Sedation and Safety During Endoscopic Procedures. 2003.

5. NCEPOD. Scoping our Practice - the 2004 Report of the National Confidential Enquiry into Patient Outcome and Death.

6. Day L, Kwon A, Inadomi J, et al. Adverse events in older patients undegoining colonoscopy: a systematic review and meta-analysis. Gastrointest Endosc 2011;74:855-96.

7. Leung FW. Is there a place for sedationless colonoscopy? J Interv Gastroenyterol 2011;1:19-22.

\section{PTU-210 YOUNG PATIENTS WITH PR BLEEDING: FLEXIBLE SIGMIOIDOSCOPY OR COLONOSCOPY?}

doi:10.1136/gutjnl-2012-302514c.210

J Dyson,* A Reddy. Gastroenterology, Queen Elizabeth Hospital, Gateshead, UK

Introduction PR bleeding is a common indication for endoscopy. Other risk factors for sinister pathology include increasing age, anaemia, change in bowel habit and family history of colorectal cancer. In our centre there is debate about whether a flexible sigmoidoscopy, vs colonoscopy, is sufficient for young patients with
PR bleeding alone. If sigmoidoscopy is sufficient it would reduce the risk patients are exposed to by full colonoscopy and the workload on the endoscopy unit. We ultimately aim to design a protocol for how to investigate PR bleeding.

Methods Retrospective review of all lower GI endoscopies done for either PR bleeding alone or in combination with another indication in 2008-2010. We reviewed patient age, indications and findings. Age groups were divided into $<45$ years or $\geq 45$ years. Indications were divided into PR bleeding alone or plus another indication.

Results 1492 procedures were done in this period. 15 were abandoned. 17 of 199 (8.5\%) procedures performed in people under 45 years for PR bleeding alone found polyps. The histology showed 10 metaplastic polyps, $1 \times 15 \mathrm{~mm}$ rectal low grade villous adenoma, $1 \times 12 \mathrm{~mm}$ sigmoid low grade tubulovillous adenoma, $1 \times 2 \mathrm{~mm}$ sigmoid low grade tubular adenoma, 1 prolapsed haemorrhoid, 1 polypoid ganglioneuroma, $1 \times 3 \mathrm{~mm}$ splenic polyp (not retrieved for histology) and $1 \times 2 \mathrm{~mm}$ sigmoid polyp not removed given current GI bleed.

Conclusion No patients in the low risk group had cancer. Only two patients $(1 \%)$ had large $(>10 \mathrm{~mm}$ ) polyps (low grade dysplasia, completely excised), both within reach of a flexible sigmoidoscope. No patients in the younger age group with PR bleeding as the sole indication would have had significant pathology (large polyp or cancer) missed due to having a flexible sigmoidoscopy rather than colonoscopy. This suggests that a protocol for this group could be implemented to prevent unnecessary tests with the associated incumbent risks. Further review of a larger cohort is required to ensure that this strategy does not expose patients to an unacceptable risk of missing significant pathology.

Abstract PTU-210 Table 1

\begin{tabular}{|c|c|c|c|c|c|c|c|}
\hline & \multicolumn{4}{|c|}{ PR bleeding only } & \multicolumn{3}{|c|}{ PR bleed + another indication } \\
\hline & $\begin{array}{l}45 \text { years } \\
(\mathrm{n}=199)\end{array}$ & \multicolumn{3}{|c|}{$\begin{array}{l}\geq 45 \text { years } \\
(n=581)\end{array}$} & $\begin{array}{l}45 \text { years } \\
(n=186)\end{array}$ & \multicolumn{2}{|c|}{$\begin{array}{l}\geq 45 \text { years } \\
(n=511)\end{array}$} \\
\hline Normal* & 127 & & 213 & & 50 & 64 & \\
\hline Inflam $†$ & 17 & & 104 & & 14 & 28 & \\
\hline Polyp & 10 & Left-10 & 48 & & 3 & 24 & \\
\hline Cancer & 0 & & 9 & Left-9 & 0 & 11 & Left-11 \\
\hline \multicolumn{8}{|l|}{ Colon } \\
\hline Normal $^{*}$ & 28 & & 59 & & 78 & 143 & \\
\hline Inflam† & 10 & & 65 & & 34 & 108 & \\
\hline Polyp & 7 & Left-7 & 76 & & 7 & 116 & \\
\hline Cancer & 0 & & 7 & Left-6, Right-1 & 0 & 17 & Left-14, Right-3 \\
\hline
\end{tabular}

Competing interests None declared.

\section{PTU-211 PATIENT PREPARATION PRIOR TO GASTROSCOPY: A UK WIDE SURVEY}

doi:10.1136/gutjnl-2012-302514c.211

J Callaghan,* J Neale, P Boger, P Patel. Gastroenterology, Southampton University Hospital, Southampton, UK

Introduction Background: Optimal patient preparation for colonoscopy is shown to improve polyp detection in the colon. In Japan, it is widely accepted practice to administer a mucolytic agent prior to gastroscopy to improve visualisation of the upper gastrointestinal tract. There is a paucity of robust UK studies which describe optimal methods of preparation prior to gastroscopy. The current variations in UK practice have not been quantified. Aims 